\title{
¿Debemos ir de la educación especial a la educación inclusiva? Perspectivas y posibilidades de avance
}

\section{Should we move from special education to inclusive education? Perspectives and possibilities of advance}

Dr. Rodolfo Cruz Vadillo es Doctor en Investigación Educativa de la Universidad Popular Autónoma del Estado de Puebla (México) (rodolfo.cruz@upaep.mx) (http://orcid.org/0000-0002-2561-1559)

Recibido: 2017-09-27 / Revisado: 2018-03-29 / Aceptado: 2018-04-30 / Publicado: 2018-07-01

\section{Resumen}

El presente trabajo es resultado de una investigación que tuvo como objetivo analizar las creencias que sobre la inclusión de personas con discapacidad tienen los profesores al atender a un estudiante en situación de discapacidad. El propósito de este texto es problematizar la idea que la educación inclusiva es un espacio discursivo y representacional que no se ha dado gracias a la transición o continuidad de la educación especial. La metodología es de corte cualitativo con alcance descriptivo desde un paradigma interpretativo. Se utilizó un cuestionario con 13 preguntas abiertas realizadas a 61 profesores de educación obligatoria todos estudiantes de la maestría en Pedagogía de una Institución de Educación Superior de la ciudad de Puebla. El tipo de análisis que se dio a los resultados fue de discurso. Los resultados señalan que a pesar de que en algunos discursos se ha mostrado una división casi antagónica, para los profesores la mixtura y complementariedad es necesaria, es decir, el saber de la educación especial complementa los principios de igualdad, equidad y no discriminación de la educación inclusiva. Las conclusio- nes a las que se llega es la necesidad de complementariedad entre la educación inclusiva y la educación especial para avanzar en un proyecto de desarrollo humano.

Descriptores: Educación inclusiva, discapacidad, discurso, educación especial, profesores, educación.

\begin{abstract}
The present work is the result of an investigation that had as objective to analyze the beliefs about the inclusion of people with disabilities in the teaching of a disabled student. The purpose of this text is to problematize the idea that inclusive education is a discursive and representational space that has been given thanks to the transition or continuity of special education. The methodology is qualitative with a descriptive scope from an interpretative paradigm. A questionnaire was used with 13 open questions made to 61 teachers of compulsory education, all students of the master in Pedagogy of a Higher Education Institution in the city of Puebla. The results were given a speech type of analysis. The results indicate that although some discourses have shown an almost antagonistic division, for
\end{abstract}

Forma sugerida de citar: Cruz, R. (2018). ¿Debemos ir de la educación especial a la educación inclusiva? Perspectivas y posibilidades de avance. Alteridad, 13(2), 251-261. https://doi.org/10.17163/alt.v13n2.2018.08. 
teachers mixing and complementing is necessary, that is, the knowledge of special education complements the principles of equality, equity and non-discrimination of the inclusive education. The reached conclusions show the need for complementarity between inclusive

\section{Introducción}

Las políticas educativas en la última década en México $^{1}$ han apuntado sus esfuerzos a nuevos procesos de subjetivación (Soriano, 2015), donde, por un momento, se ha superado una idea abstracta de persona, para dar paso a nuevas particularidades, más cercanas y regionales que permiten comprender un pequeño fenómeno desde su historicidad y contexto político, económico y social. La intención con este ejercicio es interrogar la concepción de igualdad entre los sujetos y así permitir un paso casi intersticial de la diferencia y la diversidad como aspectos constitutivos de nuestra humanidad (Gardou, 2016; Skliar, 2002).

Los conceptos de inclusión educativa (IE), educación inclusiva (EI) y persona con discapacidad (PCD), son significantes que aparecen constantemente en los discursos educativos. El objetivo último de dichas apariciones tendría que ser la configuración de prácticas discursivas materializadas en acciones educativas que permitan problematizar el espacio escolar para repensar las actuaciones, los ajustes y las identidades que se han jugado en las relaciones pedagógicas.

Aunque en este trabajo se señala el elemento discursivo, los cambios que implican no son sólo nominales o a nivel de los conceptos, parten de una reconfiguración ontológica y epistemológica sobre el accionar del docente y toda la comunidad educativa frente a la diferencia y la diversidad que hoy por hoy los nuevos ordenamientos buscan propiciar, y en algunos casos, llaman al imperativo y la obligatoriedad.

Mucho se ha escrito sobre el tema, es decir, en la literatura académica que hace referencia al espacio discursivo de lo educativo, este saber está en movimiento constante, lo cual ha dado education and special education to advance in a human development project.

Keywords: Inclusive education, disability, discourse, special education, teachers, education.

pie a diversos estudios sobre la inclusión de PCD a espacios institucionalizados como la escuela en igualdad de condiciones (Aguerrondo, 2008; Ainscow, y Miles, 2008; Arnaiz, 2012; Echeita, y Duk, 2008; Fernández, 2003; Jacobo, 2012; Juárez, Comboni, y Garnique, 2010; Ocampo, 2015; Yadarola, 2006). Sin embargo, el problema que se ha observado no tiene que ver con el número de propuestas y de elaboraciones sobre el tema, sino la forma en que el juego de las representaciones ha tomado para la constitución de algunas subjetividades.

En este trabajo se pretende problematizar el tema de la EI y su relación con la educación especial (EE), sobre todo lo que tiene que ver con la inclusión de PCD. Uno de los objetivos es señalar aquéllos aspectos que muchas veces ya no se cuestionan pues parecen estar cristalizados en el tiempo, es decir, algunos discursos que han constituido un tipo de mirada hacia lo educativo y que han quedado casi inamovibles en un determinado periodo temporal. En este sentido, se busca identificar las omisiones, exclusiones, discursos, relaciones, etc. que están presentes cuando se habla de EE y EI.

Otro de los objetivos es poner en tensión e interrogar dichos significantes con la finalidad de señalar sus distintas procedencias discursivas y epistemológicas, y los espacios de emergencia desde los cuales han aparecido. Aquí interesan los saberes que han permitido la enunciación de sus principales postulados y los efectos de verdad que han construido en los profesores de educación obligatoria en la ciudad de Puebla.

Para finalizar, se retoman los discursos de los profesores de educación obligatoria de la ciudad de Puebla, todos estudiantes de un posgrado en Pedagogía de una Institución de Educación Superior. En el análisis se intentan mostrar las 
lógicas o sistemas de razón que han permitido la construcción de un tipo de representación, el cual muchas veces no ha permitido la constitución de espacios y prácticas discursivas que beneficien los procesos de inclusión en instituciones escolares.

\section{Herramientas teórico- analíticas: presentación de una caja de herramientas}

En toda investigación la teoría juega un papel sumamente importante, pues ayuda a construir explicaciones e interpretaciones y a problematizar fenómenos, en este caso interesan los educativos (Sautu, 2003; Buenfil, 2006). En estudios de corte cualitativo, dicho elemento cobra aún mayor importancia, pues son constructos que entran en juego siempre desde la delimitación del problema a investigar, iluminando parte del fenómeno y permitiendo construir nuevo conocimiento del mismo.

La teoría, como aquí se entiende, es vista como una caja de herramientas conceptual, herramientas que dependiendo el uso que les den permitirán explicaciones e interpretaciones de una determinada realidad.

Cabe mencionar que la teoría entonces no es un ente monolítico que lo explica todo y que posee verdades totalizadoras y totalizantes, más bien nos brinda herramientas donde la utilización de las mismas recae en el investigador, a manera que es su pericia y dominio el que puede permitir o no determinada explicación; un uso que constituya conocimiento en torno a un tema específico. Partiendo de lo anterior, lo que en este trabajo se abordan como fenómenos educativos son conceptos como EE y EI, ambos relacionados con el tema de la inclusión de personas con discapacidad en las escuelas de educación obligatoria en México.

Desde esta perspectiva analítica y teórica, se habla de rescatar y analizar los discursos sobre EI y EE que circulan como referentes en las instituciones educativas y que han permeado un tipo de representación de los profesores. Cabe señalar que dichos discursos, más que meros conceptos objetivos y que muestran una realidad estable, continua y atemporal, señalan la constitución de realidades y subjetividades en lo social.

Desde la perspectiva de Foucault (1968, 2010), un discurso supera la cuestión de los conceptos, las variaciones lingüísticas e incluso, aunque reconoce los elementos extra lingüísticos, destaca esta visión señalando como unidad de análisis los enunciados.

Cabe señalar que con enunciado no se hace referencia a la sintaxis de una lengua, a la conformación de un sujeto con un predicado cuyas reglas gramaticales permitan el encadenamiento de palabras. Por enunciado se debe entender lo que en un espacio, configuración y práctica discursiva puede ser dicho. También tiene que ver con la identificación del sujeto que se ha ordenado a ese discurso y que está legitimado para enunciarlo (Foucault, 1968, 2010 y Barcalett, 2016).

Como se ha apuntado, una primera unidad de análisis para poder aproximarse a los discursos es el enunciado. Sin embargo, como ya se había mencionado, el análisis de los enunciados no está sometido sólo a una particularidad sintáctica, semántica o morfológica, cuestión tradicional en análisis lingüísticos, más bien está aparejado con un juego político y de poder- saber.

En este marco, es necesario abordar los enunciados desde otra lógica o sistema de razón ${ }^{2}$, donde lo importante no son los conceptos en sí, sino que éstos cobran significado a partir de un juego de representaciones. Es decir, desde un sentido más clásico del uso de los enunciados y los conceptos, los segundos han sido los que mayor interés han presentado a la hora de analizar determinadas estructuras lingüísticas, no obstante, este tratamiento ha traído una serie de omisiones y problemas. Uno de ellos tiene que ver con poner al concepto como la unidad principal de análisis, visión que lo mira como un objeto de uso neutro, recurso que señala una relación directa con las realidades o problemas que se quiere comprender cuando se investiga.

Desde la perspectiva epistémica de este trabajo, no hay cosa menos incierta que proceder 
de esta forma. Si se acude a la noción signo de Saussure (1945), se pueden observar las reminiscencias de este pensamiento, lógica o sistema de razón que llevó a algunos investigadores sociales a buscar la unicidad y universalidad del lenguaje, empresa hasta ahora imposible de realizar cuando se reconoce que cualquier lenguaje no señala directamente a la realidad, sino que sirve como medio y por tanto, es el uso del mismo el que permitirá o no que se construyan diferentes cuerpos teóricos sobre otros.

Si esta empresa hasta la fecha se ha tornado imposible, se tiene que intentar visualizar y problematizar, no los conceptos como unidad de análisis, sino los enunciados. El enunciado según Foucault (2010), no pretende la petrificación de significados, es más, supera las meras definiciones y amplía su margen analítico al señalar que no es tanto lo que se dice cómo quién lo dice, desde qué lugar lo dice y en qué juego de verdad y orden del discurso se encuentra el sujeto que lo enuncia.

Pero ¿cómo entonces podemos aproximarnos al análisis de los discursos? ¿Qué herramientas teóricas pueden ayudarnos a comprender el orden que guía a los discursos? ¿Cómo realizar una interpretación no literal, ahistórica y atemporal? Foucault (1988) propone un tipo de analítica genealógica que permite dicha aproximación.

La genealogía o el análisis genealógico de Foucault (1988) es una crítica a una comprensión histórica de los fenómenos que premia la unidad del sujeto en torno a un origen, una universalidad y una continuidad de los fenómenos. Menciona que desde un sistema de razón donde existe un sustento epistemológico que resalta una idea de positividad de los sucesos y la continuación en el tiempo, no permite observar los espacios más regionales, las rupturas, las exclusiones, las luchas de poder, los enfrentamientos por el saber.

Los hechos entonces deben ser abordados desde su genealogía, desde la lógica del acontecimiento, desde un juego de emergencia y procedencia, donde el origen, el centro, la estructura construida de los sucesos no existe como tal. Más bien está lo discontinuo, lo histórico, las microsituaciones, etc.

El acontecimiento entonces señala el espacio discursivo en el cual determinado enunciado puede ser dicho, un contexto de enunciación complejo que ha permitido la constitución de verdades en lo social (Foucault, 1988). Luego entonces, la lógica que guía a los fenómenos o discursos como acontecimientos no permite pensar la continuidad, es decir, dicho discurso no está sujeto a la lógica de causa y efecto, más bien al accidente.

Es así que podemos decir que emergen en un tiempo y espacio determinado, pero también en un juego de verdad que permite su enunciación, la construcción de un enunciado que puede ser aceptado como verdadero. La situación es que dicha emergencia no tiene un origen unificado, más bien procede del resultado de configuraciones y prácticas que le permitieron, por vía de la vecindad, el encuentro y el parentesco, emerger. La procedencia también pensada como accidente, no causal, no acumulativa, sino espontánea y muchas veces impredecible (Foucault, 1988).

\section{La educación especial y la educación inclusiva: ¿dónde colocar a las personas con discapacidad?}

Gran parte de la literatura de actualidad aborda el tema de la educación especial y educación inclusiva desde la lógica de la continuidad, no es necesario entrar de lleno a todos los enunciados que se emiten, basta ver los títulos principales, los que lógicamente funcionan también como enunciados. Enunciados que anuncian el paso de la EE a la EI en esta idea lineal y continua de un discurso a otro (Juárez, Comboni, y Garnique, 2010; Parra, 2011; de León, 2010).

El problema con este tipo de explicaciones es que no permiten comprender los sistemas de razón y lógicas de intelección que se encuentran atrás de los conceptos. El uso actual de los mis- 
mos está pensado como un fin en sí mismos. Las últimas reformas han puesto el tema como imperativo desde los ordenamientos, han solicitado un paso o cambio de paradigma, pero poco se han preocupado por explicar cómo se ha constituido la aparente revolución educativa y también científica. Por ejemplo, la idea de que hay que pasar de un modelo de EE a uno de EI (SEP, 2017) está siendo diseminada como un discurso fuerte que anuncia una relación de alteridad y por ende un tipo de antagonismo ${ }^{3}$ que señala la casi extinción de un elemento frente al otro.

La EE, en su paso por los discursos educativos y pedagógicos tuvo un lugar de emergencia y una procedencia muy distinta a la EI. Como acontecimiento, la educación especial parte de una visión individual y biomédica del sujeto (Skrtic, 1996; Warner, 1996; De la Vega, 2010). El pathos está presente desde todo su fundamento epistemológico. Se podría entender más bien una cuestión de salud, un tipo de ortopedia sobre los cuerpos que busca sanar.

La emergencia tiene que ver con la generalización de una abstracción universalizante de lo humano, de un sujeto. Un tipo de igualación formal que tuvo un pilar importante con el cogito cartesiano (Barcalett, 2016). La existencia dentro de la humanidad de una racionalidad inherente a todos propició pensar en la intervención de aquellos que aparentemente no se encontraban en esa posibilidad.

La cuestión tenía que ver con una mirada hacia la normalización de las acciones de la capacidad individual, hacia el camino en construcción de una imagen de hombre/mujer abstracto, iguales, altamente racionales. La entrada de la ciencia biomédica y la biopolítica jugó un papel central. Los estudios médicos sobre los seres humanos pronto devinieron en intervenciones de los cuerpos de los sujetos considerados "anormales" (Foucault, 2014). Sujetos que muchas veces sólo se encontraban en un estadio de atraso, por lo cual la intervención ortopédica de redireccionamiento y compostura debía ser el camino (función psi). La procedencia de estos discursos y la capacidad de construir enunciados viene desde la mirada médica, de la enfermedad, del pathos sobre el cuerpo que hay que curar.

Si analizamos entonces y comparamos el acontecimiento de la educación inclusiva veremos no es un agregado de educación especial, que no es un mejoramiento de la misma, que no es su continuidad y por ende el estadio al que determinada positividad nos debe llevar.

La educación inclusiva como acontecimiento emerge desde un lugar de enunciación diferente, no parte de estudios "científicos" ni su mirada tiene que ver con un proceso de individuación, aquí no hay nada que sanar.

Más bien su procedencia se puede colocar en otra práctica discursiva, en otra configuración. Apunta más bien a los espacios, en lugar que al cuerpo, se aleja del pathos y señala un sujeto que más que ser algo patológico, es jurídico. Su espacio discursivo lo encuentra en la ley, en los principios de justicia, que lejos están de poseer una mirada individual sobre el cuerpo que hay que reparar.

La interrogación de la EI no es al sujeto sino a los enunciados que han permitido construir unas subjetividades en lo social, donde algunos han sido excluidos. Un juego de inclusión y exclusión.

Como se puede observar los sistemas de razón son diametralmente opuestos en muchos de sus elementos. Por un lado desde la propia concepción de sujeto, por otro, los mecanismos mediante los cuales un enunciado se ha considerado como verdadero, es decir, la constitución de saberes que en un determinado tiempo y espacio dieron cuenta de una subjetividad desde un pathos o desde una visión de derecho.

Ocampo (2015), señala a la EI más como un movimiento político, hacer EI o propiciar la inclusión tiene que ver con un juego de fuerzas políticas y no tanto con la configuración de la $\mathrm{EE}$ como saber.

La EI no se origina o no procede del conocimiento de la EE, tampoco interroga dicho conocimiento. Su lugar es otro muy diferente, y consis- 
te en la reconfiguración de los espacios simbólicos desde los cuales nosotros interactuamos día a día. En este marco, el conocimiento de la EE no va a ser sustituido por el conocimiento de la EI pues no existe una relación causal entre los mismos.

Poner a debatir a nivel epistémico dichos conceptos no está llevando a la solución de las problemáticas. Se tiene que aceptar la coexistencia no antagónica de los saberes que constituyen los enunciados de cada discurso. Pensar cómo su convivencia puede coadyuvar tanto a la autorrealización de las personas en su individualidad y también a la creación de espacios que permitan a esas personas la capacidad de articular una serie de funcionamientos (Nussbaum, 2007, 2012).

El problema en este punto no es que se haya construido un discurso lineal y de estadios en torno a la educación especial e inclusiva, más bien la cuestión radica en la escasa posibilidad de éxito en la relación antagónica establecida. Es decir, el conocimiento de la educación especial es necesario para establecer una educación inclusiva. Los antagonismos creados no ayudan porque constituyen la extinción de una para la existencia de la otra.

El peligro recae en la pertinencia y éxito de ambas configuraciones discursivas desde esta lógica binaria que no permite pensar lo complejo de la realidad y la complementariedad discursiva. En este sentido, se tendría que caminar hacia la constitución de enunciados que reconstituyan la interacción dialógica de ambos discursos, los cuales desde su posición presentan una posibilidad como juego de verdad y de lenguaje propios.
Aquí puede aplicar la lógica de arriba hacia abajo y abajo hacia arriba. El lugar de encuentro tendría que ser el contexto del sujeto visto como un todo y no la decisión de una u otro.

\section{Método}

Esta investigación tuvo un alcance descriptivo e interpretativo. El instrumento para la recolección de datos fue un cuestionario cualitativo de 13 preguntas abiertas. Los sujetos del estudio fueron 61 profesores de educación obligatoria de la ciudad de Puebla y estudiantes de un posgrado (Ver tabla 1). Los datos fueron transcriptos cuidando la fidelidad de los mismos para poder realizar un análisis de discurso.

Se realizó un proceso de codificación que consistió en lo siguiente: como eran alumnos de posgrados se procedió a colocar una "A"; una "U" que señalaba su estatus de universitario y posteriormente el número de cuestionario que les fue asignado de acuerdo a la forma en que se recogieron los mismos.

Los cuestionamientos que se pensaron para el instrumento tuvieron como objetivo central: "Analizar las creencias que sobre la inclusión de personas con discapacidad tienen los profesores al atender a un estudiante en situación de discapacidad", no obstante, para este texto sólo se problematiza el discurso que habla de un paso de la educación especial a la educación inclusiva". Para ellos se pensaron las dimensiones expuestas en la siguiente tabla. (Ver tabla 2):

Tabla 1. Dimensiones e ítems

\begin{tabular}{|l|l|}
\hline \multicolumn{2}{|c|}{ Dimensión } \\
\hline $\begin{array}{l}\text { Implicaciones y condiciones } \\
\text { del trabajo al atender a estu- } \\
\text { diantes con discapacidad }\end{array}$ & $\begin{array}{l}\text { - Trabajar con un alumno con discapacidad implica. } \\
\text { - Las condiciones con las que debe contar la institución para trabajar con un alumno } \\
\text { con discapacidad son. }\end{array}$ \\
\hline $\begin{array}{l}\text { Aspectos que implican la inclu- } \\
\text { sión de estudiantes con disca- } \\
\text { pacidad al aula regular desde } \\
\text { la práctica docente }\end{array}$ & $\begin{array}{l}\text { - Las fortalezas, beneficios o aspectos positivos que tiene el trabajar con un estu- } \\
\text { diante con discapacidad son. }\end{array}$ \\
\hline
\end{tabular}




\begin{tabular}{l|l}
$\begin{array}{l}\text { Sentimientos, valores y acti- } \\
\text { tudes que se presentan en el } \\
\text { trabajo con estudiantes con } \\
\text { discapacidad. }\end{array}$ & $\begin{array}{l}\text { - Cuando un profesor trabaja con un alumno con discapacidad se manifiestan va- } \\
\text { lores como. } \\
\text { - Al trabajar con un alumno con discapacidad las actitudes que están presentes en } \\
\text { el docente son. }\end{array}$ \\
\hline $\begin{array}{l}\text { Concepto y creencias de edu- } \\
\text { cación especial, inclusiva y dis- } \\
\text { capacidad. Conflictos éticos de } \\
\text { los docentes. }\end{array}$ & $\begin{array}{l}\text { - La educación inclusiva hace referencia a. } \\
\text { - La discapacidad es. }\end{array}$ \\
$\begin{array}{l}\text { - La educación especial hace referencia a. } \\
\text { - El lugar idóneo para que los alumnos con discapacidad aprendan es. } \\
\text { - Los conflictos éticos a los que se enfrentan los docentes cuando trabajan con } \\
\text { alumnos con discapacidad son. }\end{array}$
\end{tabular}

\section{Fuente: elaboración propia}

Por cuestiones de espacio, los resultados que se presentan aquí sólo corresponden a la dimensión "Concepto y creencias de educación especial, inclu- siva y discapacidad. Conflictos éticos de los docentes", el ítem analizado fue "El lugar idóneo para que los alumnos con discapacidad aprendan es".

Tabla 2. Caracterización de los participantes

\begin{tabular}{|l|l|}
\multicolumn{1}{|c|}{ Nivel educativo } & \multicolumn{1}{|c|}{ Número de docentes } \\
\hline Educación Básica & 47 \\
\hline Educación Media Superior & 14 \\
\hline
\end{tabular}

\section{Resultados: ¿es el paso de la educación especial a la educación inclusiva un siguiente estadio? Algunos datos a partir de los docentes}

En lo referente al tema del paso de la EE a la $\mathrm{EI}$, aspecto que ya es señalado en México como una condición de paso de modelo a otro con la finalidad de constituir espacios más justos, equitativos y menos discriminatorios, es uno de los puntos que se han abordado en el ejercicio de cuestionario con los profesores, sujetos de este estudio.

Para ellos existen tres posibilidades de dar atención a alumnos con discapacidad. Una de ellas tiene que ver con que ellos estén en la EE, donde se les puede dar una atención adecuada.
AU61 "Hay profesores capacitados y cuentan con instalaciones adecuadas"

AU46 "En ese espacio hay personal especializado en el tema, además las aulas y los materiales son para uso exclusivo de personas con discapacidad"

AU45 "Los docentes regulares no están capacitados en primeros auxilios"

AU22 "La atención a ese tipo de alumnos no se puede dar en la escuela regular pues nosotros docentes no estamos capacitados como los de educación especial, sobre todo para atender la sordera, los invidentes, etc."

AU23 "Porque se necesita una infraestructura especial que cobra un aporte positivo para atender a las discapacidades"

Según los comentarios anteriores podemos identificar dos aspectos principales del por qué la EE es el lugar para las personas con discapacidad. 
En este sentido, conviene recordar que las ideas aquí expuestas representan la condición del enunciado el cual encierra una representación de lo que en determinado orden discursivo puede ser dicho.

En un primer momento aparece el tema de los apoyos técnicos y la infraestructura, es decir, las escuelas de educación especial presentan mejores condiciones de accesibilidad y adaptaciones que son imprescindibles para atender a alumnos con discapacidad. Hay un fuerte componente centrado en el espacio que es un condicionante para que se ejerza un tipo de autonomía en los estudiantes.

Por otro lado aparece otro tema que al parecer es muy diferente al primero. Mientras que en lo referente a la infraestructura no se señala más que aspectos materiales que tienen su correlato con las inversiones económicas que implican realizar los ajustes en las instituciones y que son parte de los objetivos de las luchas por una EI, en este segundo punto aparece la cuestión del saber de la EE.

Para los profesores el saber de la EE supera la mera visión pedagógica de la misma, es decir, se enfrentan a un componente que está lejos de su labor docente, que tiene su génesis desde un paradigma del pathos, de la enfermedad, de la mirada médica y clínica. Si bien, los compañeros no han expresado el concepto que tienen sobre la discapacidad y las personas con discapacidad, en la pregunta por el espacio se sospecha la comorbilidad existente entre un ser humano y una condición deficitaria que lo reduce a esta segunda situación. Una finitud donde lo patológico es la base para las decisiones sobre las personas, en este caso sobre el lugar que deben ocupar en el campo social. No obstante la evidencia de esta emergencia y su procedencia, no se puede negar que dichas configuraciones discursivas, a modo de un saber especializado, son una condición necesaria que permite la comprensión de fenómenos relacionados con la situación deficitaria de algunas PCD por tanto hoy por hoy, estas ideas representan enunciados que desde un sistema de razón son válidos.
Por otro lado están aquéllos que señalan al aula regular como el espacio idóneo para las PCD. Aquí los anunciados abordan, desde otro sistema de razón, aquello que es prudente decir, pero frente a una procedencia diferente, una visión de derecho y más social.

AU54 "Contextualiza a la persona en una sociedad real"

AU40 "El aprendizaje colectivo es más significativo y da mejores resultados"

AU43 "Los alumnos pueden desarrollarse plenamente con la sociedad y alumnos y niños de su edad, dando mayor seguridad a los niños"

AU3 "Cuando el alumno termine de ir al aula, se enfrentará a la vida real y no hay modos especiales, mejor ir desarrollando otras habilidades que le sirvan en un aula regular"

AU49 "Pues porque es un derecho, porque no debería existir exclusión"

AU8 "Porque si están en la escuela especial no van a aprender cómo relacionarse con todo en la vida real, las dificultades se encuentran en el día con día, en la calle"

AU31 "Ya que con el apoyo de los compañeros el alumnos con discapacidad podría desarrollar otras habilidades, además de que sería incluirlo en la sociedad"

Como se puede observar, las explicaciones de los profesores que apoyan la atención de estudiantes con discapacidad para que ésta sea llevada a cabo en el aula regular no difieren significativamente, ponen en el centro lo social, una participación sin exclusión, incluso, como ellos lo llaman, "el encuentro con un mundo real", donde todos conviven en un mismo espacio, lo cual es un derecho humano y fundamental.

$\mathrm{Si}$ se comparan ambas posturas, no se puede decir que alguna carece de un cierto efecto de verdad, que hay errores insuperables en las explicaciones, pues ambas parten de un sistema lógico que es válido y señalan diversos y diferentes elementos que desde la literatura son fundamentales cuando se habla de un desarrollo humano. 
Por un lado, está la parte de poder desarrollar capacidades sustantivas y combinadas (Nussbaum, 2007, 2012), es decir, que se adquieran habilidades y destrezas que permitan a la persona poder estar en el mundo de forma autónoma y con autodeterminación. Por otro lado, está otra capacidad que tiene que ver con la oportunidad de poder desplegar funcionamientos en espacios que no hagan diferencias. Es decir, desde lo que Nussbaum $(2007,2012)$ defiende en su enfoque de capacidades, que se desarrolle el ser humano en lo individual pero que a su vez se construya el escenario idóneo para ello.

Es así como ambas posturas más que ser opuestas, podrían resultar complementarias. Así lo ha expresado otro grupo de profesores, el mayor en esta investigación.

AU13 "Porque se realizaría un trabajo en equipo, en donde el docente de aula regular sabrá cómo trabajar con apoyo y se realiza la inclusividad"

AU36 "Para complementar una con la otra"

AU2 "Al saber y sentirse integrado en un grupo social motiva al alumno a desarrollar habilidades y destrezas. Además el docente debe tener un conocimiento especial para brindar las correctas herramientas"

AU18 "Porque a veces en la escuela regular los docentes carecemos de los conocimientos para atender de la mejor manera a estos alumnos, pero el que asistan a la escuela regular los apoya a sentirse mejor y aprender"

AU58 "Creo que las dos brindan apoyo en el alumno y se fortalecen entre las dos para beneficiarlo"

AU52 "Son necesarias porque consideran entonces la inclusión y la equidad"

AU48 "Se requiere de personas capacitadas que atiendan a fondo las necesidades, pero que también el alumno sea integrado a la sociedad"

AU42 "Ambas, porque necesitan convivir y aprender de los niños regulares y contar con un apoyo individual para el proceso de enseñanza”
AU59 "El alumno con discapacidad debe estar en el aula regular y compartir experiencias, sin embargo, puede asistir a recibir apoyo complementario a una escuela de educación especial"

En los discursos políticos y educativos se han puesto en contraposición la EE de la EI, como ya se había mencionado en líneas anteriores, su genealogía es distinta, no emergieron desde una relación antagónica, es decir, el antagonismo que se ha proliferado no es tal en el sentido que la presencia de una, arriesga la existencia de la otra.

En los comentarios anteriores se puede señalar dicha complementariedad en dos elementos. En un primero, la necesidad de un conocimiento especializado que puede favorecer en lo individual la capacidad de las personas y por otro la construcción de una capacidad combinada, es decir, la constitución de un espacio que permita la interacción de funcionamientos, la participación, la autonomía y la autodeterminación.

En línea con lo anterior, crear una capacidad en donde los funcionamientos y distintos tipos de capacidades puedan estar en un mismo espacio. El problema desde los discursos que se han manifestado y el uso que le hemos dado a los mismos, es que han imaginado una relación antagónica entre dos sistemas de razón que no reclaman la aniquilación del otro, sino el trabajo en equipo. Si como ya se había apuntado, la genealogía de la EE, su origen, el espacio y sinergia de fuerzas que la hicieron acontecer, muy lejos está de ser el inicio o continuación de la EI. No hay una revolución científica al estilo de Kuhn (1945), donde un saber debe o puede desaparecer con la presencia de ciencia nueva.

Lo que los comentarios señalan es la convivencia de dos espacios discursivos a modo de prácticas que deben coexistir en un mismo lugar para poder lograr el desarrollo de lo humano en las PCD. Entonces no es cuestión de decidir el lugar dónde estarán, necesitamos, como lo menciona Echeita (2014), pensar en cómo vamos a dar respuesta a sus necesidades y para eso nece- 
sitamos toda la ayuda posible, desde los saberes que se han constituido.

\subsection{Conclusiones}

Los enunciados que se analizaron en este trabajo y que señalan algunas configuraciones discursivas son fruto de una serie de enfrentamientos de fuerza a nivel discursivo. Como se señaló, existe un interés político y educativo por el tema de la inclusión y la educación de las PCD. Y ha sido desde este componente político donde se ha dado lugar al conflicto entre dos posturas, que en el espacio simbólico y representacional, se han opuesto como un par binario. En este texto se ha intentado explicar que, desde un análisis genealógico, dicha disposición es inexistente.

La propuesta fue describir un poco las lógicas y sistemas de razón que han dado paso a la emergencia y acontecimiento de ambos modelos educativos. Aquí se ha señalado que su procedencia es diferente y que en realidad el antagonismo es inexistente y además imposibilita la empresa inclusiva.

Desde los enunciados y discursos de los profesores, se ha dado este enfrentamiento, hay algunos que han sido interpelados y han tomado postura, sin embargo sus explicaciones no son contradictorias, es decir, los que han optado por una u otra opción no han podido señalar la constitución de un par oposicional; más bien han señalado elementos constitutivos de cada discurso, demostrando más la relación de alteridad, la complementariedad de los mismos.

No obstante lo anterior, la mayoría de los profesores han enunciado este elemento que representa un tipo de mixtura, una convivencia pacífica que representa la complejización del espacio más que la simplificación desde cada discurso.

La afrenta desde esta tercer vía no facilita el problema, más bien anuncia la necesidad de nuevas configuraciones discursivas desde las políticas educativas que no simplifiquen ambos discursos (EE y EI) sino que, desde una postura crítica, coadyuven a la generación de capacidades donde se permita cualquier tipo de funcionamiento. La pregunta ya no debe ser por el espacio, sino por la creación de una comunidad que aprende y se desarrolla como un todo sistémico.

\section{Referencias bibliográficas}

Aguerrondo, I. (2008). Revisar el modelo: un desafío para evitar la exclusión. Perspectivas Dossier: Educación Inclusiva, 38(1), 61-80. Recuperado de https://goo.gl/Ub3bwf

Ainscow, M. y Miles, S. (2008). Por una educación para todos que sea inclusiva: ¿Hacia dónde vamos ahora? Perspectivas Dossier: Educación Inclusiva. 38(1), 14-44. Recuperado de https://goo.gl/wRMYyA.

Arnaiz, P. (2012). Cómo avanzar para que la educación inclusiva llegue a todos. En Z. Jacobo, S. Vargas y L. Meléndez (Comps.), Sujeto, educación especial e integración (pp. 1-20). México: UNAM.

Barcalett, M. (2016). Una historia de la anormalidad. Finitud y ciencias en la obra de Michel Foucault. México: Editorial Gedisa y UAEM.

Buenfil, R. N. (1994). Cardenismo. Argumentación $y$ antagonismo en educación. México, DIE Cinvestav/ Conacyt.

Buenfil, R. N. (2006). Usos de la teoría en la investigación educativa. En M. Jiménez (Coord.), Los usos de la teoría en la investigación (pp. 37-59). México: Plaza y Valdés.

Cruz-Pineda, O. (2012). El uso teórico en el estudio de las políticas educativas. En R.N. Buenfil, S. Fuentes S. y E. Treviño (Coords.), Giros teóricos II. Diálogos y debates en las ciencias sociales y humanidades (pp. 159-172). México: FFL- UNAM.

De la Vega, E. (2010). Anormales, deficientes y espaciales. Genealogía de la educación especial. Buenos Aires: Noveduc.

De León, N. (2010). De la educación especial al paradigma de la cultura de la diversidad y la educación inclusiva. El caso del Instituto Herbert. (Tesis de maestría). Universidad Internacional de Andalucía, España.

Echeita, G. (2014). Educación para la inclusión o educación sin exclusiones. Madrid: Nárcea. 
Echeita, G. y Duk, C. (2008). Inclusión Educativa. REICE, Revista Electrónica Iberoamericana sobre Calidad, Eficacia y Cambio en Educación (2), 1-8.

Fernández, A. (2003). Educación inclusiva: Enseñar y aprender entre la diversidad. Revista Digital UMBRAL 2000, 13, 1-10. Recuperado de https://goo.gl/kXeuZq

Foucault, M. (1968). Las palabras y las cosas. México: Siglo XXI Editores.

Foucault, M. (1988). Nietzsche, la genealogía y la historia. Valencia: Pre- textos.

Foucault, M. (2010). La arqueología del saber. México: Siglo XXI Editores.

Foucault, M. (2014). Los anormales. México: Fondo de Cultura Económica.

Gardou, Ch. (2016). Nadie tiene la exclusividad del patrimonio humano y social. En P. Brogna, Z. Jacobo y R. Cruz (Coords.), Voces de la alteridad (pp. 19-30). México: UNAM.

Jacobo, Z. (2012). ¿De qué ética hablamos de la de inclusión o de la diferencia? En Z. Jacobo, S. Vargas y L. Meléndez (Comp.), Sujeto, Educación Especial e Integración (pp. 47-66). México: UNAM.

Juárez, J., Comboni, S., \& Garnique, F. (2010). De la educación especial a la educación inclusiva. Argumentos, 3(62), 41-83. Recuperado de https://goo.gl/TftahS

Kuhn, J. S. (1971). La estructura de las revoluciones científicas. México: Fondo de Cultura Económica.

Nussbaum, M. (2007). Las fronteras de la justicia. Barcelona: Paidós.

Nussbaum, M. (2012). Crear capacidades: propuesta para el desarrollo humano. Barcelona: Paidós

Ocampo, A. (2015). Aproximaciones y descripciones generales sobre la Formación del Objeto de la Educación Inclusiva. En A. Ocampo (Coord.), Los rumbos de la educación inclusiva en Latinoamérica en los inicios del siglo XXI. Cartografías para modernizar el enfoque (pp. 24-90). Santiago de Chile: Celei.

Parra, C. (2011). Educación Inclusiva: un modelo de diversidad humana. Revista Educación y Desarrollo Social, 1, 139- 159.

Saussure, F. (1945). Curso de lingüística general. Buenos Aires: Editorial Losada.
Sautu, R. (2003). Todo es teoría. Objetivos y métodos de investigación. Buenos Aires: Lumiere

Secretaría de Educación Pública (2017). El Modelo Educativo 2017. Recuperado de https://goo.gl/KkvXza

Skliar, C. (2002) Alteridades y pedagogías. O... ¿y si el otro no estuviera ahí? Educação \& Sociedade. 23(79), 85-123. Recuperado de https://goo.gl/ zvKByK

Skrtic, T. (1996). La crisis en el conocimiento de la educación especial: Una perspectiva sobre la perspectiva. En B. Franklin (Comp.), Interpretación de la discapacidad. Teoría e historia de la educación especial (pp. 35-72). Barcelona: Ediciones Pomares.

Soriano, R. (2012). Algunas herramientas conceptuales para comprender la constitución de sujetos educativos. En O. Cruz Pineda (Coord.), Investigación y teoría. Tensiones y rejuegos (pp. 57-71). México: Ed. Sequitur/ PAPDI.

Warner, M. (1996). Realismo crítico como una metateoría para la educación especial. En B. Franklin (Comp.), Interpretación de la discapacidad. Teoría e historia de la educación especial (pp.73-89). Barcelona: Ediciones Pomares.

Yadarola, M. (2006). Una mirada desde y hacia la Educación Inclusiva. Boletín Electrónico de IntegraRed. Recuperado de https://goo.gl/Xij2Cv

\section{Notas}

1 Programa Nacional de Fortalecimiento de la Educación Especial y la Integración Educativa (2000); Ley de Personas con discapacidad (2005); Orientaciones para el funcionamiento de los servicios de educación especial (2006); Programa Sectorial de Educación (2007, 2010); Ley General para la Inclusión de personas con discapacidad (2011); Modelo Educativo para la Educación Obligatoria (2017).

2 Cruz- Pineda (2012, p.169) señala que los sistemas de razón "son sistemas de conocimiento que a través de los cuales se establecen nuevas representaciones sociales, es decir, el individuo construye nuevas y diferentes subjetividades por medio de las cuales establecen nuevas y diferentes relaciones no sólo entre los sujetos sino también con el conocimiento".

3 Buenfil, (1994, p. 19) conceptualiza el antagonismo como "un vínculo que se establece entre dos subjetividades que se niegan recíprocamente. Se diferencia de contradicción y de oposición real por un lado y de subordinación, dominación y opresión, por el otro lado. El antagonismo no alude al plano de lo empírico, sino al orden simbólico, donde se establecen las relaciones sociales como tales." 